\title{
The 2D dynamics of the differentially rotating envelope of massive stars
}

\author{
Delphine Hypolite ${ }^{1}$, Stéphane Mathis ${ }^{1}$ and Michel Rieutord ${ }^{2}$ \\ ${ }^{1}$ Laboratoire AIM Paris-Saclay, CEA/DRF - CNRS - Université Paris Diderot, IRFU/SAp \\ Centre de Saclay, F-91191 Gif-sur-Yvette Cedex, France \\ email: delphine.hypolite@cea.fr \\ ${ }^{2}$ Institut de Recherche en Astrophysique et Planétologie, Observatoire Midi-Pyrénées, \\ Université de Toulouse, 14 avenue Edouard Belin, 31400 Toulouse, France
}

We build a 2D model of the radiative envelope of main sequence massive stars. We set a dynamical boundary condition at the bottom of the radiative envelope at $\eta=r_{C} / R$ (where $r_{C}$ is the core size and $R$ the radius of the star) to account for the differential rotation of the convective core as computed in 3D simulations (e.g. Browning et al. (2004, IAUS, 224, 149). We seek the differential rotation and associated meridional circulation induced by such a shear competing with the baroclinic flow of the stably stratified radiative envelope using the Boussinesq approximation. Our study shows that the resulting dynamics depends on the Rossby number $\mathcal{R} o=\Delta \Omega / 2 \Omega_{0}$, (e.g. Brown et al. (2008, ApJ, 689, 1354), Augustson et al. (2012, ApJ, 756, 169), Varela et al. (2016, ASR, 58, 1507)) which is the ratio of the differential rotation in the convective core over the global stellar rotation rate. When the Rossby number is higher than one, the differential rotation has a columnar structure as dictated by the Taylor-Proudman theorem, as illustrated in Fig. 1, coming from the shear applied by the boundary condition. The associated circulation does not develop much in the envelope leading to a quasi solid rotation outside the tangent to the core cylinder. When it is lower than one the dynamics is driven by the baroclinic torque. The meridional circulation redistributes angular momentum in the entire envelope through viscosity and leads to a differential rotation with a rapid pole and a slow equator (e.g. Rieutord, 2006, A\&A, 451, 1025).
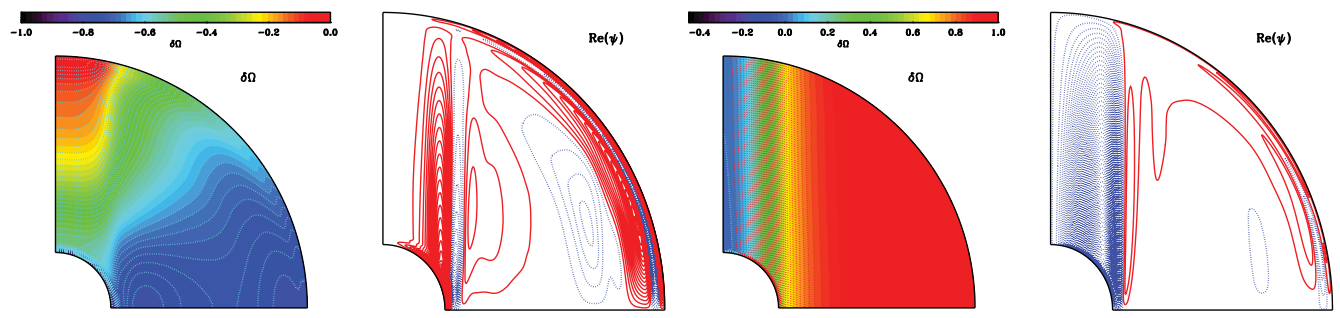

Figure 1. Left: Differential rotation $\Delta \Omega$ (relative to the pole rotation) and associated meridional circulation stream function $\Psi$ (red: direct sens) in the radiative envelope, when $E=10^{-5}$ and $\mathcal{R} O=10^{-1}$. The rotation axis is vertical. Right: Same for $E=10^{-5}$ and $\mathcal{R} o=10$. 